\title{
Subcutaneous Injection of Large Volumes of Polidocanol
}

Sclerotherapy is a standard treatment of varicose veins and telangiectasias. ${ }^{1}$ Different sclerosing agents have been used, with sodium tetradecyl sulfate (STS) and polidocanol (POL) being the most frequent molecules. Both solutions are detergents and may be emulsified to produce foam, which will increase the potency over 2fold and decrease toxicity 4 -fold.

The risk of local complications after injection of POL seems relatively low. Cutaneous ulcerations have been reported between $0.2 \%$ and $1.2 \%$ of cases. The authors here report the case of a patient who was erroneously administered a high volume of POL as local anesthetic with no major and long-lasting complication.

\section{Observation}

A 19-year-old female patient presented with a pigmented lesion on her right forearm. On clinical examination and dermoscopy, the lesion showed some atypical features and was therefore excised to rule out melanoma. The patient was subcutaneously injected with a 7-mL solution of an anesthetic thought to represent lidocaine plus epinephrine. Because no significant anesthetic and vasoconstrictor effect was observed after 10 minutes, the physician finally realized to have erroneously used a $0.5 \%$ POL solution instead and canceled the planned excision after informing the patient about the mistake. There were no acute local or systemic adverse effects. Over the following days, the patient developed a slight painful and warm local tumefaction at the site of injection. The ultrasound examination revealed a subcutaneous edematous swelling (Figure 1). At no point did the patient or physician observe any loss of subcutaneous tissue or signs of fat atrophy. The lesion was finally excised 6 weeks later and light microscopy studies showed the presence of a dysplastic melanocytic nevus with an underlying moderate, lobular panniculitis. There was no evidence for either necrosis or vascular occlusion (Figure 2).

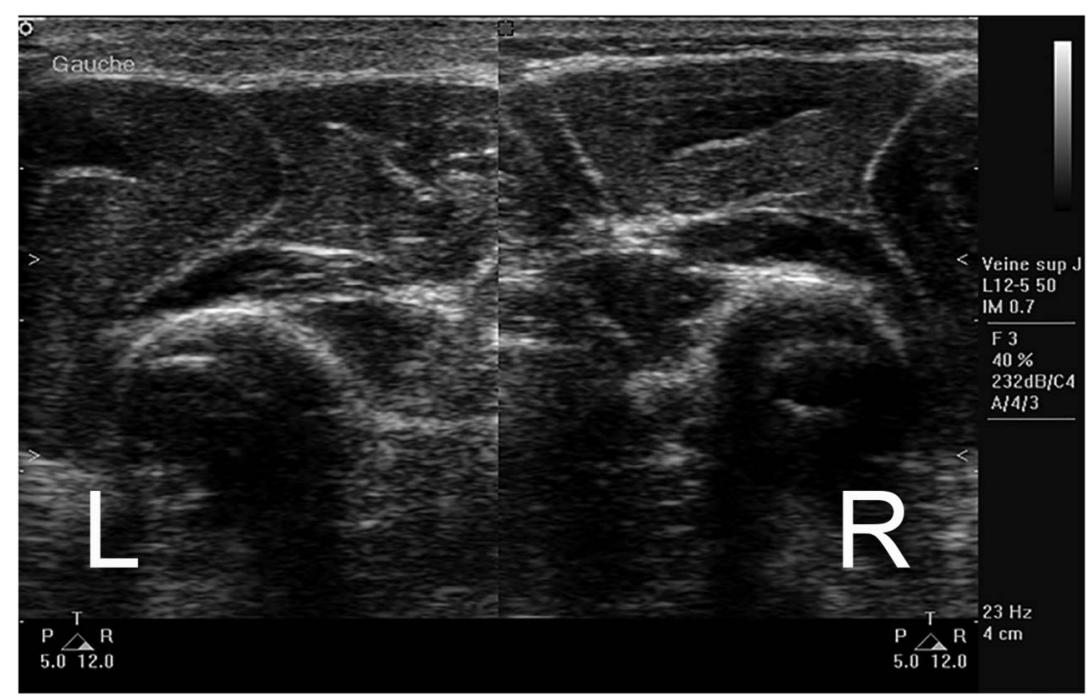

Figure 1. Transversal sonographic image of the left (L) and right (R) forearms, 2 weeks after injection of $7 \mathrm{~mL}$ of $0.5 \%$ polidocanol showing an edematous, subcutaneous swelling on the right forearm.

(c) 2018 by the American Society for Dermatologic Surgery, Inc. Published by Wolters Kluwer Health, Inc. All rights reserved. ISSN: 1076-0512 - Dermatol Surg 2018;0:1-3• 

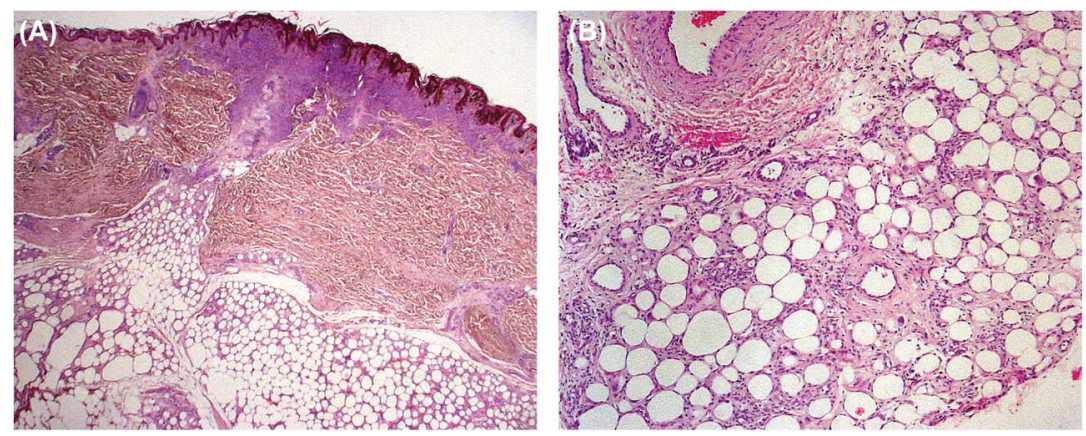

Figure 2. Dysplastic melanocytic nevus (A) with an underlying panniculitis but no tissue necrosis (B) 4 weeks after subcutaneous injection of $7 \mathrm{~mL}$ of $0.5 \%$ polidocanol.

\section{Discussion}

Tissue necrosis may occur after sclerotherapy with various sclerosing agents. ${ }^{1}$ This complication is rare and typically observed with caustic solutions such as hypertonic saline. The exact mechanisms responsible for cutaneous necrosis remain unclear. A variety of factors have been discussed such as direct toxic effects of the extravasated molecules, vascular occlusion due to intra-arteriolar injection, reactive vasospasm, or injection of arteriovenous shunts, which may variably result in tissue hypoxia and tissue destruction. ${ }^{1}$

Polidocanol, which was introduced in 1936 as a local and topical anesthetic, ${ }^{1}$ is still used today in some dermatological and proctological creams and ointments. Previous experimental studies have shown that subcutaneous injection of POL may lead to damage of muscle cells of the tunica intima and media of the blood vessels as well as, to some extent, myelinated axons of the paravascular nerves. ${ }^{1,2}$ However, the surrounding tissues did not seem to be significantly damaged. These observations contributed to the commercialization of POL as a sclerosing agent. Other reports underlined the safety of POL up to $3 \%$, even after extravascular injection in rats and in rabbits. ${ }^{3,4}$ In humans, local edema was observed after 4 accidental injections of POL (1-2 mL, 0.5\%-2\%), without late complications (Kreussler Pharmaceuticals, unpublished data). ${ }^{1}$ However this risk seems to be different with STS as showed by Duffy, where deliberate mid-dermal injection of 0.4 $\mathrm{mL}$ of $3 \%$ POL did not induce tissue necrosis, but 0.4 $\mathrm{mL}$ of $1 \%$ STS did. ${ }^{1}$
In the authors' patient, infiltration with $7 \mathrm{~mL}$ of $0.5 \%$ of POL resulted in a moderate swelling and localized painful sensation. Although a clinical and histological panniculitis was visible, no damage or necrosis of dermal vessels and the subcutaneous tissue was observed.

Nonetheless, the effects of higher concentrations of POL extravasate are more unpredictable. The authors observed the development of a severe Nicolau dermatitis 4 days after ultrasound-guided sclerotherapy with $4 \mathrm{~mL}$ of $2 \%$ POL foam. ${ }^{1}$ This delayed reaction might be due to an arterial vasospasm after POL's transparietal diffusion. The POL-related vessel wall damage after paravasal injection has also been therapeutically used, such as for the management of red telangiectasias ("Quaddeln") ${ }^{1}$ and anterior epistaxis. ${ }^{5}$ The effects of these procedures may be related to the toxic effect of POL while diffusing through the vessel walls.

Even if accidental paravasal injection may be well tolerated, it is important that the managing physician is familiar with the injection techniques and carefully considers the available guidelines to limit the risk of iatrogenic complications.

\section{References}

1. Goldman MP, Weiss RA. Treatment of Varicose and Telangiectatic Leg Veins (6th ed). Edinburgh, United Kingdom: Elsevier; 2017.

2. Staubesand J, Cheon MJ. Spätschäden an peripheren Nerven durch Paravasate von Polidocanol-eine elektronenmikroskopische Studie. Phlebologie 1994;23:112-9. 
3. Sato D, Kurita M, Ozaki M, Kaji N, et al. Extravascular injection of sclerotic agents does not affect vessels in the rat: experimental implications for percutaneous sclerotherapy of arteriovenous malformations. Eur J Vasc Endovasc Surg 2012;44:73-6.

4. Schuller-Petrovic S, Pavlovic MD, Neuhold N, Brunner F, et al. Subcutaneous injection of liquid and foamed polidocanol: extravasation is not responsible for skin necrosis during reticular and spider vein sclerotherapy. J Eur Acad Dermatol Venereol 2011;25: 983-6.

5. Farneti P, Pasquini E, Sciarretta V, Macri G, et al. Comparison of local sclerotherapy with lauromacrogol versus nasal packing in the treatment of anterior epistaxis. Clin Exp Otorhinolaryngol 2016;9: $131-5$.
FABIAN SCHIBLER, MD

LUCA BORRADORI, MD

Albert-Adrien Ramelet, MD

Department of Dermatology, Inselspital

Bern University Hospital

University of Bern

Bern, Switzerland

The authors have indicated no significant interest with commercial supporters. 